\section{¿Afecta la forma de puntuación la estructura factorial del GHQ-12? Estudio exploratorio en estudiantes iberoamericanos}

\author{
Does the GHQ-12 scoring system affect its \\ factor structure? An exploratory study of \\ Ibero American students
}

\begin{abstract}
A forma de pontuação do GHQ-12 afeta o fator de estrutura do instrumento? Estudo exploratório sobre estudantes ibero-americanos
\end{abstract}

\begin{abstract}
This study aimed to evaluate whether the scoring system of the General Health Questionnaire (GHQ-12) alters the instrument's factor structure. The method considered 1,972 university students from nine Ibero American countries. Modeling was performed with structural equations for 1, 2, and 3 latent factors. The mechanism for scoring the questions was analyzed within each type of structure. The results indicate that models with 2 and 3 factors show better goodness-of-fit. In relation to scoring mechanisms, procedure 0-1-1-1 for models with 2 and 3 factors showed the best fit. In conclusion, there appears to be a relationship between the response format and the number of factors identified in the instrument's structure. The model with the best fit was 3-factor 0-1-1-1-formatted, but 0-1-2-3 has acceptable and more stable indicators and provides a better format for two- and three-dimensional models.
\end{abstract}

Questionnaires; Statistical Factors Analysis; Statistical Models
Alfonso Urzúa 1 Alejandra Caqueo-Urízar 2 Mariana Bargsted 1 Matías Irarrázaval 3

\section{Resumen}

El objetivo de este estudio fue evaluar si el sistema de puntuación del Cuestionario de Salud General (GHQ-12) puede alterar la estructura factorial del instrumento. El método utilizado consideró a 1.972 estudiantes universitarios, correspondientes a 9 países iberoamericanos. Se realizó un modelamiento con ecuaciones estructurales para 1, 2, y 3 factores latentes. Dentro de cada tipo de estructura se analizó el mecanismo de puntuación de las preguntas. Los resultados indican que los modelos de 2 y 3 factores presentan mejores indicadores de bondad de ajuste. En relación a los mecanismos de puntuación, el procedimiento 0-1-1-1 para los modelos de 2 y 3 factores presenta mejor ajuste. En conclusión, pareciera existir una relación entre el formato de respuesta y el número de factores identificados en la estructura del instrumento. El modelo que muestra mejor ajuste entre todos es el de 3 factores con formato 0-1-1-1, sin embargo, el formato 0-1-2-3 tiene indicadores aceptables y más estables, siendo un mejor formato para modelos bi y tridimensionales.

Cuestionarios; Análisis Factorial; Modelos Estadísticos 


\section{Introducción}

El Cuestionario de Salud General (GHQ) fue elaborado como un instrumento de autoreporte, diseñado para el tamizaje de trastornos psíquicos o distrés psicológico, en un ámbito comunitario o en medios clínicos no psiquiátricos. Ha sido ampliamente utilizado y, como resultado, traducido a varios idiomas 1,2 .

El instrumento ha sido objeto de una serie de modificaciones desde el GHQ original, que comprende 60 elementos, creándose versiones más abreviadas, como la de 12 ítems (GHQ-12). Esta última adaptación fue traducida y adecuada a diversos idiomas y goza de una gran popularidad en cuanto a su uso. Esto se debe, principalmente, a su buen funcionamiento y reducida extensión, reportándose propiedades psicométricas adecuadas, mostrando validez y confiabilidad satisfactorias y presentando, además, una consistencia interna que va de un rango de 0,80 a 0,90 dependiendo del estudio 3,4. En cuanto a la correlación de cada uno de los ítems con los demás, se han observado valores de correlación superiores a 0,51. El GHQ-12 muestra una elevada consistencia interna en población general y también en población mayor de 65 años 5 .

Pese a lo extendido de su uso, existen dos grandes dilemas aún no resueltos, en cuanto a su utilización. Por una parte, se ha asumido una estructura unidimensional, por otra, existen diversos sistemas de puntuación de la escala.

Sobre la estructura factorial, aun cuando la propuesta inicial de Goldberg \& Hillier ${ }^{6}$ para la versión de 12 afirmaciones correspondía a un factor, diversas investigaciones han reportado que este supuesto no se estaría cumpliendo. En ellas se explora la existencia de diferentes factores o dimensiones teóricas del instrumento, describiendo la presencia de una, dos y tres dimensiones 5 .

Para explorar la dimensionalidad del GHQ12 se ha utilizado el análisis de sus componentes principales, identificándose una estructura de dos factores. El primero corresponde al factor “disforia general”, que consiste en 7 ítems relacionados con ansiedad y depresión. Un segundo factor de "disfunción social" que consiste en 6 ítems relacionados con actividades diarias y habilidades de enfrentamiento de situaciones difíciles 7 .

Se ha propuesto modelos alternativos de 2 factores. Uno de ellos consiste en 6 ítems del factor "ansiedad/depresión", 5 ítems del factor “actividades diarias" y "desempeño social”, y el ítem "no me puedo concentrar", separado de los dos factores anteriores 8. Otro modelo encontró evidencias para un factor "depresión” con 4 ítems y “disfunción sexual” con 3 ítems 9 .

Hasta la fecha no se ha precisado la existencia de una o más estructuras factoriales del GHQ12 en población general, aunque sí se ha visto una mejor distribución de las dimensiones del cuestionario, dependiendo de las características de la muestra en estudio. Es así como diversos autores sugieren estudiar más a fondo la estructura factorial del GHQ-12 y cómo su utilización, basada en una o más dimensiones, se ajusta de mejor manera a la población objetivo de cada grupo de investigación. En apoyo a lo anterior, se plantea que los niveles de angustia de una población pueden afectar la estructura factorial del GHQ-12. Esto ayudaría a explicar por qué las estructuras factoriales han sido tan diversas en diferentes poblaciones. Los estudios que investigan la interacción entre sesgos cognitivos y ansiedad han demostrado que los individuos ansiosos muestran un sesgo en el procesamiento de la información, prestando atención preferente a estímulos amenazantes y ambiguos 1,3,10.

Por otra parte, en relación con los sistemas de puntuación del GHQ-12, éstos pueden ser de tipo ordinal (0-1-2-3) o binarios (0-0-0-1, 0-0-1-1, 0-11-1). Sin embargo, en una muestra adulta francesa, se ha encontrado que la escala de tipo ordinal o Likert produce una distribución más aceptable de los resultados para llevar a cabo análisis paramétricos (con menos sesgo y menos curtosis) donde un mayor puntaje indica un mayor grado de angustia. Cada elemento del cuestionario se califica en una escala de cuatro puntos. Un grupo de investigadores utilizó este tipo de puntuación con modelos de dos y tres factores, observando que se ajustaban muy bien a los datos y con buena consistencia interna, mientras que con el modelo de un solo factor no ocurrió lo mismo. Se obtuvo un alfa de Cronbach de 0,78 para el modelo unidimensional, mientras que para los modelos de dos y tres factores el alfa de los valores resultó ser: ansiedad/depresión $=0,84$; disfunción social $=0,76$; pérdida de confianza $=0,817$. Otros autores sugieren que, para fines estadísticos, este tipo de puntuación sería el más apropiado 11. Asimismo, para una muestra de adolescentes españoles, las respuestas también se asignaron en una escala Likert de 4 puntos, con la misma puntuación utilizada por Salama-Younes et al. 12 .

La Tabla 1 muestra los resultados de diversos estudios en relación a los factores encontrados, así como su sistema de puntuación.

Actualmente, no se ha encontrado reporte acerca de si el sistema de puntuación utilizado podría alterar la estructura factorial de la escala, convirtiéndose esta interrogante en nuestra pregunta de investigación. 
Características de los diversos modelos factoriales y sistemas de puntuación para el Cuestionario de Salud General (GHQ-12).

\begin{tabular}{|c|c|c|c|c|c|}
\hline Modelos & $\begin{array}{l}\text { Número } \\
\text { de factores }\end{array}$ & Factor 1 & Factor 2 & Factor 3 & $\begin{array}{l}\text { Sistema de } \\
\text { puntuación }\end{array}$ \\
\hline Worsley \& Gribbin 23 (1977) & 3 & $2,5,6,7,9$ & $1,3,4,8,12$ & 10,11 & $0-1-2-3$ \\
\hline Golberg \& Williams 24 (1988) & 1 & $\begin{array}{c}1,2,3,4,5,6,7,8,9,10 \\
11,12\end{array}$ & & & $0-0-1-1$ \\
\hline Greatz 17 (1991) & 3 & $2,5,6,9$ & $1,3,4,7,8,12$ & 10,11 & $0-1-2-3$ \\
\hline Kilic et al. 25 (1997) & 2 & $2,4,5,6,9,10,11$ & $1,3,7,8,12$ & & $0-0-1-1$ \\
\hline Politi et al. 7 (1994) & 2 & $2,5,6,9,10,11,12$ & $1,3,4,7,8,12$ & & $0-1-2-3$ \\
\hline Martin 26 (1999) & 3 & $1,3,4,8,11$ & $2,5,7$ & $6,9,12$ & $1-5$ \\
\hline Doi \& Minowa 27 (2003) & 3 & $2,5,6,9,10$ & $1,3,4,7,8$ & 3,12 & $0-0-1-1$ \\
\hline Kalliath et al. 28 (2004) & 2 & $4,7,8,12$ & $6,9,10,11$ & & $0-5$ \\
\hline $\begin{array}{l}\text { Andrish \& van Schoubroeck } 29 \\
\text { (1989) }\end{array}$ & 2 & $1,3,4,7,8,12$ & $2,5,6,9,10,11$ & & $0-0-1-1$ \\
\hline Schmitz et al. 8 (1999) & 2 & $1,2,3,6,7,10,11$ & $3,4,5,8,9,12$ & & $0-0-1-1 / 0-1-2-3$ \\
\hline Hakins ${ }^{1}$ (2008) & 1 & $\begin{array}{c}1,2,3,4,5,6,7,8,9,10 \\
11,12\end{array}$ & & & $\begin{array}{c}0-0-1-1 / 0-1-1-1 / \\
0-1-2-3\end{array}$ \\
\hline Montazari et al. 30 (2003) & 2 & $1,3,4,7,8,10,11$ & $2,5,6,9,12$ & & $0-0-1-1$ \\
\hline Shevlin \& Adamson 31 (2005) & 3 & $2,5,6,9$ & $1,3,4,7,8,12$ & 10,11 & $0-1-2-3$ \\
\hline Guerje 32 (1991) & 2 & $3,6,7,9,11,12$ & $1,2,4,5,7,10$ & & $0-1-2-3$ \\
\hline Picardi et al. 33 (2001) & 3 & $2,10,11,12$ & $3,4,5$ & $7,8,9$ & $0-1-2-3$ \\
\hline Daradkeh et al. 34 (2001) & 3 & $5,6,7,8,9$ & $1,3,4,11,12$ & $2,9,10$ & $0-1-2-3$ \\
\hline Farrell 35 (1998) & 3 & $2,10,11,12$ & $1,6,7,8,9$ & 3,4 & $0-0-1-1$ \\
\hline Campbell \& Knowles 36 (2007) & 1 & $\begin{array}{c}1,2,3,4,5,6,7,8,9,10 \\
11,12\end{array}$ & & & $0-1-2-3$ \\
\hline $\begin{array}{l}\text { Sánchez-López \& Dresch } 15 \\
\text { (2008) }\end{array}$ & 3 & $1,3,4,7,8,12$ & $6,9,10,11$ & $2,5,9$ & $0-1-2-3$ \\
\hline Salama-Younes et al. 12 (2009) & 3 & $1,3,4,7,8,12$ & $2,5,6,9$ & 10,11 & $0-1-2-3$ \\
\hline Li et al. (2009) 22 & 2 & $1,3,4,7,8,12$ & $2,5,6,9,10,11$ & & $0-1-2-3$ \\
\hline Padrón et al. 4 (2012) & 3 & $2,5,6,9$ & $1,3,4,7,8,12$ & 10,11 & $0-1-2-3$ \\
\hline Gouveia et al. 37 (2012) & 1 & $\begin{array}{c}1,2,3,4,5,6,7,8,9,10 \\
11,12\end{array}$ & & & $1-4$ \\
\hline Rey et al. 38 (2014) & 1 & $\begin{array}{c}1,2,3,4,5,6,7,8,9,10 \\
11,12\end{array}$ & & & $\begin{array}{c}0-0-1-1 / 0-1-1-1 / \\
0-1-2-3\end{array}$ \\
\hline Molina et al. 39 (2014) & 1 & $\begin{array}{c}1,2,3,4,5,6,7,8,9,10 \\
11,12\end{array}$ & & & $0-1-2-3$ \\
\hline Aguado et al. 40 (2012) & 1 & $\begin{array}{c}1,2,3,4,5,6,7,8,9,10 \\
11,12\end{array}$ & & & $0-1-1-1$ \\
\hline
\end{tabular}

El objetivo de este estudio es evaluar si la forma de puntuación del GHQ-12 afecta la estructura factorial del cuestionario.

\section{Método}

\section{Participantes}

Fueron evaluadas 1.972 personas, correspondientes a 9 países iberoamericanos. Los partici- pantes corresponden a una muestra intencionada de estudiantes de diversas carreras de educación universitaria en las ciudades de Antofagasta (Chile), Lima (Perú), La Habana (Cuba), San José (Costa Rica), Ciudad de México (México), Asunción (Paraguay), Jujuy (Argentina), Bogotá (Colombia) y Barcelona (España). 
Instrumento

El GHQ-12 corresponde a un cuestionario autoadministrado, dirigido a la situación actual del encuestado (Tabla 2). Es una versión abreviada del original, siendo ampliamente utilizado para medir el bienestar psíquico en algunos trastornos mentales e identificar la severidad de trastornos psiquiátricos menores. Comparativamente con sus otras versiones, ha demostrado igual utilidad según diferentes estudios de validación efectuados 13,14.

Este cuestionario ha sido adaptado y ampliamente utilizado en España 15 y también ha sido objeto de adaptación y validación en Chile 16 . La escala usada por cada ítem es tipo Likert con puntuaciones que van de 0 a 3 , midiendo dos áreas, salud general y funcionamiento social. La consistencia interna del GHQ ofrece un coeficiente alfa de Cronbach de 0,89 12.

\section{Procedimiento}

Una vez obtenida la aprobación del Comité de Ética de la Universidad Católica del Norte, en Chile, se procedió a las coordinaciones necesarias con los distintos miembros del Grupo Interuniversitario de Investigaciones en Psicología de la Salud, a fin de organizar el proceso de levantamiento de datos. Los cuestionarios fueron pasados en las respectivas aulas de clases, previa firma de un consentimiento informado. Los datos se recogieron entre los meses abril y noviembre del 2010. Se aplicaron aproximadamente 2.300 encuestas, se respondieron satisfactoriamente 1.972. Se consideraron como criterios de exclusión la discapacidad cognitiva o visual, que dificultara la correcta cumplimentación del instrumento. Los análisis fueron realizados a través del programa estadístico SPSS 17 (SPSS Inc., Chicago, EEUU) y EQS para Windows (Multivarinate Software Inc., Encino, EEUU).

Para alcanzar los objetivos de investigación se realizó un modelamiento con ecuaciones estructurales para 1, 2 y 3 factores latentes. Dentro de cada tipo de estructura se analizó el mecanismo de puntuación de las preguntas.

Los modelos analizados para cada forma de puntuación (0-1-2-3; 0-0-0-1; 0-0-1-1; 0-1-1-1) fueron el unidimensional (preguntas 1 a la 12), bidimensional (factor 1 con preguntas 1, 3, 4, 7, 8,12 y factor 2 con preguntas $2,5,6,9,10,11$ ) y la propuesta de tres dimensiones elaborada por Graetz 17 (factor 1 ansiedad y depresión, preguntas 2, 5, 6 y 9; factor 2 anhedonia y disfunción social, preguntas 1, 3, 4, 7, 8 y 12; factor 3 pérdida de confianza y autoestima, preguntas 10 y 11), lo que equivale a un total de 12 modelos a evaluar.
Tabla 2

Ítems del Cuestionario de Salud General (GHQ-12).

\begin{tabular}{|c|c|}
\hline Número & Ítem \\
\hline 1 & ¿Ha podido concentrarse bien en lo que hace? \\
\hline 2 & $\begin{array}{l}\text { ¿Sus preocupaciones le han hecho perder } \\
\text { mucho sueño? }\end{array}$ \\
\hline 3 & $\begin{array}{l}\text { ¿Ha sentido que está jugando un papel útil en } \\
\text { la vida? }\end{array}$ \\
\hline 4 & ¿Se ha sentido capaz de tomar decisiones? \\
\hline 5 & $\begin{array}{l}\text { ¿Se ha sentido constantemente agobiado y en } \\
\text { tensión? }\end{array}$ \\
\hline 6 & $\begin{array}{l}\text { ¿Ha sentido que no puede superar sus } \\
\text { dificultades? }\end{array}$ \\
\hline 7 & $\begin{array}{l}\text { ¿Ha sido capaz de disfrutar sus actividades } \\
\text { normales de cada día? }\end{array}$ \\
\hline 8 & $\begin{array}{l}\text { ¿Ha sido capaz de hacer frente a sus } \\
\text { problemas? }\end{array}$ \\
\hline 9 & ¿Se ha sentido poco feliz y deprimido? \\
\hline 10 & ¿Ha perdido confianza en sí mismo? \\
\hline 11 & $\begin{array}{l}\text { ¿Ha pensado que usted es una persona que } \\
\text { no vale para nada? }\end{array}$ \\
\hline 12 & $\begin{array}{l}\text { ¿Se siente razonablemente feliz considerando } \\
\text { todas las circunstancias? }\end{array}$ \\
\hline
\end{tabular}

Para comparar los modelos que estaban anidados se utilizó la prueba de diferencia de chicuadrado Satorra-Bentler.

Los principales índices de ajuste del modelo utilizados son el índice de ajuste no normado de Bentler y Bonnet (NFI) y el índice de ajuste comparado (CFI). En estos índices utilizados, en general los valores superiores a 0,95 son indicativos de un buen ajuste entre el modelo hipotetizado y el empírico.

Existen diferentes sugerencias en la literatura sobre el número, tipo y los valores de corte para la bondad del ajuste requerido, orientado al análisis factorial confirmatorio 18. Para el contraste del modelo estructural se contemplaron tres índices de bondad de ajuste absoluto: (1) el error medio cuadrático de aproximación a valores de la población (RMSEA), cuyo valor ideal es $\leq 0,5$; (2); el índice de bondad de ajuste de Joreskog (GFI), el cual se interpreta como una proporción de varianza explicada análoga a $\mathrm{R}^{2}$ en regresión múltiple, por lo que un GFI de 1,0 indicaría ajuste perfecto, lo cual significa que el modelo explica el $100 \%$ de la varianza en los datos observados. Cuanto más próximo a 1 sea el valor, mejor bondad de ajuste en este indicador. El criterio más comúnmente aceptado es de $\geq 0,90$; (3) el índice de bondad ajustado de Joreskog (AGFI), que 
ajusta el GFI tomando en cuenta los grados de libertad en el modelo a prueba. El criterio que se asigna regularmente a este indicador es $\geq 0,9018$.

Para la estimación de los parámetros del modelo se utilizó el método de estimación más ampliamente empleado, el que corresponde al de máxima verosimilitud (maximum likelihood). Se realizó un análisis la matriz de correlaciones policóricas, lo que permite obtener una modificación "robusta" del método de estimación de máxima verosimilitud.

\section{Resultados}

Del total de sujetos evaluados, el $63 \%$ fueron mujeres. La edad promedio correspondió a 21 años. La Tabla 3 describe a los participantes por país, sexo y promedio de edad.

En la Tabla 4 se presentan las estadísticas de bondad de ajuste para los tres modelos y los cuatro tipos diferentes de puntuación. Los valores de chi-cuadrado en todos los análisis fueron significativos $(\mathrm{p}<0,01)$.

Tabla 3:

Descripción de los participantes.

\begin{tabular}{lccccc}
\hline País & Frecuencia & Hombres & Mujeres & Edad promedio & DE \\
\hline Costa Rica & 285 & 97 & 187 & 21,30 & 3,05 \\
Perú & 140 & 43 & 97 & 18,94 & 1,53 \\
México & 198 & 97 & 101 & 19,87 & 2,18 \\
Cuba & 170 & 91 & 79 & 121,81 & 4,76 \\
Paraguay & 191 & 69 & 84 & 21,54 & 3,46 \\
Argentina & 155 & 71 & 130 & 22,84 & 4,72 \\
Colombia & 187 & 57 & 182 & 22,86 & 3,82 \\
España & 226 & 32 & 250 & 20,13 & 3,06 \\
Chile & 420 & 168 & 1.232 & 21,21 & 2,14 \\
Total & 1.972 & 725 & & 3,40 \\
\hline
\end{tabular}

DE: desviación estándar.

Indicadores de bondad de ajuste para diversos modelos con distintos sistemas de puntuación.

\begin{tabular}{|c|c|c|c|c|c|c|c|c|}
\hline $\begin{array}{l}\text { Factores/ } \\
\text { Puntuación }\end{array}$ & $\chi^{2}$ & $\chi^{2 / g . l .}$ & CFI & $\mathrm{NFI}$ & GFI & AGFI & RMRS & RMSEA \\
\hline \multicolumn{9}{|l|}{1} \\
\hline $0-0-0-1$ & 668,303 & $668,03 / 54=12,37$ & 0,801 & 0,788 & 0,946 & 0,922 & 0,055 & $0,077(0,072-0,082)$ \\
\hline $0-0-1-1$ & 673,083 & $673,083 / 54=12,46$ & 0,878 & 0,869 & 0,935 & 0,906 & 0,009 & $0,054(0,072-0,083)$ \\
\hline $0-1-1-1$ & 1109,30 & $1109,308 / 54=20,54$ & 0,649 & 0,638 & 0,896 & 0,850 & 0,088 & $0,101(0,096-0,106)$ \\
\hline $0-1-2-3$ & 1033,69 & $1033,693 / 54=19,14$ & 0,839 & 0,832 & 0,899 & 0,854 & 0,097 & $0,097(0,092-0,103)$ \\
\hline \multicolumn{9}{|l|}{2} \\
\hline $0-0-0-1$ & 520,745 & $520,745 / 53=9,82$ & 0,848 & 0,834 & 0,958 & 0,939 & 0,047 & $0,068(0,063-0,073)$ \\
\hline $0-0-1-1$ & 534,622 & $534,622 / 53=10,08$ & 0,905 & 0,896 & 0,948 & 0,923 & 0,049 & $0,069(0,064-0,074)$ \\
\hline $0-1-1-1$ & 401,969 & $401,969 / 53=7,58$ & 0,884 & 0,869 & 0,966 & 0,950 & 0,043 & $0,059(0,053-0,064)$ \\
\hline $0-1-2-3$ & 605,039 & $605,039 / 53=11,41$ & 0,909 & 0,902 & 0,941 & 0,914 & 0,031 & $0,074(0,068-0,079)$ \\
\hline \multicolumn{9}{|l|}{3} \\
\hline $0-0-0-1$ & 484,698 & $484,698 / 51=9,50$ & 0,859 & 0,846 & 0,961 & 0,941 & 0,045 & $0,067(0,061-0,072)$ \\
\hline $0-0-1-1$ & 469,366 & $469,366 / 51=9,20$ & 0,918 & 0,909 & 0,956 & 0,933 & 0,047 & $0,065(0,060-0,071)$ \\
\hline $0-1-1-1$ & 324,791 & $324,791 / 51=6,36$ & 0,909 & 0,894 & 0,973 & 0,959 & 0,038 & $0,053(0,047-0,058)$ \\
\hline $0-1-2-3$ & 524,538 & $524,38 / 51=10,28$ & 0,922 & 0,915 & 0,952 & 0,926 & 0,050 & $0,070(0,064-0,075)$ \\
\hline
\end{tabular}

AGFI: índice de bondad ajustado de Joreskog; CFI: índice de ajuste comparado; GFI: índice de bondad de Joreskog; NFI: índice de ajuste normado de Bentler y Bonnet; RMRS: Standardized Root Mean Residual; RMSEA: Root Mean Square Error of Approximation. 
$\mathrm{Al}$ analizar el ajuste de los modelos asociados a la cantidad de factores, podemos advertir que los modelos de 2 y 3 factores presentan los mejores indicadores de bondad de ajuste. En cuanto a los mecanismos de puntuación, se aprecia que presenta mejor ajuste el procedimiento 0-1-1-1 para los modelos de 2 y 3 factores, no así para el modelo de 1 factor, donde tiene mejor ajuste el formato 0-0-1-1.

En ninguno de los modelos evaluados la razón chi-cuadrado/grados de libertad es menor que el valor esperado de 3 , siendo los valores que más se alejan los de las puntuaciones 0-1-1-1 y 0-1-2-3. Se espera que los indicadores de ajuste CFI, NFI, GFI y AGFI, sean iguales o mayores a 0,95 , valor que no es alcanzado en ninguno de los modelos evaluados, siendo los que más se acercan a este valor los de las puntuaciones 0-0-1-1 y 0-0-0-1. En el indicador RMRS (Standardized Root Mean Residual) -el cual se espera sea próximo a cero-, el valor menor y por ende expresión de un mejor ajuste de los datos, corresponde al tipo de puntuación 0-0-1-1, al igual que el valor del RMSEA (Root Mean Square Error of Approximation), donde se espera un valor menor que 0,08 .

Los resultados de los dos mejores modelos del análisis factorial confirmatorio del cuestionario de salud general GHQ-12 se presentan en la Tabla 5 .

Tabla 5

Análisis factorial confirmatorio del Cuestionario de Salud General (GHQ-12).

\begin{tabular}{lcc}
\hline & Modelo 2 & Modelo 3 \\
\hline$\chi^{2}$ & 401,969 & 524,538 \\
Df & 53 & 51 \\
P-value & $<0,01$ & $<0,01$ \\
RMSEA (IC90\%) & $0,059(0,053-0,064)$ & $0,070(0,064-0,075)$ \\
CFI & 0,966 & 0,922
\end{tabular}

CFI: índice de ajuste comparado; Df: grados de liberdad; IC90\%: intervalo de 90\% de confianza; RMSEA: Root Mean Square Error of Approximation.

\section{Discusión}

En el caso de los participantes de este estudio, se puede comprobar que existen diferencias en el reporte de enfermedad crónica entre los países participantes. Los países que reportan una mayor prevalencia de enfermedad crónica son Cuba (26,5\%) y Perú (15\%), y aquellos que poseen el reporte más bajo de enfermedad crónica provienen de México $(4,6 \%)$ y Colombia (7\%).

Como se puede observar en los resultados, pareciera existir una relación entre el formato de respuestas y el número de factores identificados en la estructura del instrumento.

Podemos señalar, además, que el modelo que muestra mejor ajuste entre todos (considerando los indicadores más altos) es el de 3 factores con formato $0-1-1-1$, seguido de 2 factores con mismos formatos. Sin embargo, el formato $0-1-2-3$ tiene indicadores más que aceptables y más estables. Es decir, con diferentes indicadores alcanza niveles altos, considerando los puntos de corte recomendados 19. En consecuencia, nos parece que sería el mejor formato para modelos bi y tridimensionales.

En este grupo de participantes, se aprecia que es el modelo 0-1-2-3 el que mejor ajusta a modelos de dos y tres dimensiones, en la línea de lo planteado en los antecedentes teóricos. Es importante mencionar que esto está apoyado en la idea de que este sería el formato que funciona mejor con población sana. Aun así, no está claro que este formato sea significativamente mejor que el 0-1-1-1, que cuenta con indicadores específicos de bondad de ajuste más alto. En definitiva, serían estos los mejores formatos al igual que lo sugerido por Campbell et al. 11 y SalamaYounes et al. 12 .

Si bien la evaluación psicométrica del GHQ12 sugiere que es un instrumento válido y confiable para medir morbilidad psiquiátrica, el examen de la estructura de factores ha llevado en varias ocasiones a la conclusión de que el GHQ12 mide la morbilidad psiquiátrica en más de un dominio 20. En cuanto al número de factores, a partir de la teoría, vemos que éstos también son sensibles al formato de puntuación, donde pareciera que dos o tres factores reflejarían mejor el constructo en este grupo poblacional. Esto es consistente con la literatura, que ha mostrado que el modelo multidimensional posee un mejor ajuste que el de un factor, además de ser el modelo que posee el mejor soporte factorial para el GHQ-12 20. Los hallazgos de este estudio son congruentes con estudios previos 4,21,22. 


\section{Resumo}

O objetivo deste estudo foi avaliar se a pontuação do Questionário de Saúde Geral (GHQ-12) pode alterar o fator de estrutura do instrumento. O método utilizado considerou 1.972 universitários correspondentes a nove países ibero-americanos. Realizou-se modelagem com equações estruturais para 1, 2 e 3 fatores latentes. O mecanismo de pontuação das perguntas foi analisado dentro de cada tipo de estrutura. Os resultados indicam que os modelos de 2 e 3 fatores apresentam os melhores indicadores de bondade de ajuste. Em relação aos mecanismos de pontuação, o procedimento 0-1-1-1 para os modelos de 2 e 3 fatores apresenta melhor ajuste. Em conclusão, parece que há uma relação entre o formato de resposta e o número de fatores identificados na estrutura do instrumento. O modelo que apresenta melhor ajuste é o de 3 fatores com formato $0-1-1-1$, no entanto, o formato 0-1-2-3 tem indicadores aceitáveis e mais estáveis, sendo mais indicado para modelos bi e tridimensionais.

Questionários; Análise Fatorial; Modelos Estatísticos

\section{Colaboradores}

A. Urzúa participó de la concepción y proyecto, recolección, análisis e interpretación de los datos, redacción del artículo y fue responsable por todos los aspectos del trabajo. A. Caqueo-Urízar colaboró en el análisis de datos y redacción del artículo y aprobación final de la versión que debe ser publicada. M. Bargsted participó del análisis de datos, redacción del artículo y revisión crítica relevante del contenido intelectual. M. Irarrázaval colaboró en el análisis de datos, redacción del artículo, revisión crítica relevante del contenido intelectual y aprobación final de la versión que debe ser publicada.

\section{Agradecimientos}

Este estudio ha sido financiado por el Convenio de Desempeño UTA-MINEDUC.

\section{Conflicto de interés}

No existe.

\section{Referencias}

1. Hankins M. The reliability of the twelve-item general health questionnaire (GHQ-12) under realistic assumptions. BMC Public Health 2008; 8:355.

2. Baumann M, Meyers R, Le Bihan E, Houssemand C. Mental health (GHQ12; CES-D) and attitudes towards the value of work among inmates of a semi-open prison and the long-term unemployed in Luxembourg. BMC Public Health 2008; 8:214.

3. Smith A, Oluboyede Y, West R, Hewison J, House A. The factor structure of the GHQ-12: the interaction between item phrasing, variance and levels of distress. Qual Life Res 2013; 22:145-52.

4. Padrón A, Galán I, Durbán M, Gandarillas A, Rodríguez-Artalejo $\mathrm{F}$. Confirmatory factor analysis of the General Health Questionnaire (GHQ-12) in Spanish adolescents. Qual Life Res 2012; 21:1291-8.

5. Rocha K, Pérez K, Rodríguez-Sanz M, Borrell C, Obiols J. Propiedades psicométricas y valores normativos del General Health Questionnaire (GHQ12) en población general española. Int J Clin Health Psychol 2011; 11:125-39.

6. Goldberg DP, Hillier VF. A scaled version of the General Health Questionnaire. Psychol Med 1979; 9:139-45.

7. Politi P, Piccinelli M, Wilkinson G. Reliabity, validity \& factor structure of the 12-item General Health Questionnaire among young males in Italy. Acta Psychiatr Scand 1994; 90:432-7.

8. Schmitz N, Kruse J, Heckrath C, Alberti L, Tress W. Diagnosing mental disorders in primary care: the General Health Questionnaire (GHQ) and the Symptom Check List (SCL-90) as screening instruments. Soc Psychiatry Psychiatr Epidemiol 1999; 34:360-6.

9. Werneke U, Goldberg DP, Yalcin I, Üstün BT. The stability of the factor structure of the General Health Questionnaire. Psychol Med 2000; 30:823-9.

10. Brosan L, Hoppitt L, Shelfer L, Sillence A, Mackintosh B. Cognitive bias modification for attention and interpretation reduces trait and state anxiety in anxious patients referred to an out-patient service: results from a pilot study. J Behav Ther Exp Psychiatry 2011; 42:258-64.

11. Campbell A, Walker J, Farrell G. Confirmatory factor analysis of the GHQ-12: can I see that again? Aust N Z J Psychiatry 2003; 37:475-83.

12. Salama-Younes M, Montazeri A, Ismaïl A, Roncin C. Factor structure and internal consistency of the 12-item General Health Questionnaire (GHQ12) and the Subjective Vitality Scale (VS), and the relationship between them: a study from France. Health Qual Life Outcomes 2009; 7:22.

13. Jacobsen B, Hasvold T, Hoyer G, Hansen V. The General Health Questionnaire. How many items are really necessary in population surveys. Psychol Med 1995; 25:957-61.

14. Goldberg D, Hillier V. A scaled version of the General Health Questionnaire. Psychol Med 1979; 9:139-45.

15. Sánchez-López M, Dresch V. The 12-Item General Health Questionnaire (GHQ-12): reliability, external validity and factor structure in the Spanish population. Psicothema 2008; 20:839-43. 
16. Trucco M, Larraín S, Campusano M. Estudio de un cuestionario para detectar desórdenes emocionales: validación preliminar. Rev Chil Neuro-Psiquiatr 1979; 17:20-6.

17. Graetz B. Multidimensional properties of the General Health Questionnaire. Soc Psychiatry Psychiatr Epidemiol 1991; 26:132-8.

18. Marsh HW, Hau K, Wen Z. In search of golden rules: comment on hypothesis testing approaches to setting cut-off values for fit indexes and dangers in over generalizing $\mathrm{Hu}$ and Bentler's findings. Structural Equation Modelling 2004; 11:320-41.

19. Clark LA, Watson D. Constructing validity: basic issues in objective scale development. Psychol Assess 1995; 7:309-19.

20. Martin CR, Newell RJ. Is the 12-item General Health Questionnaire (GHQ-12) confounded by scoring method in individuals with facial disfigurement? Psychol Health 2005; 20:651-9.

21. Li W, Chung J, Chui M, Chan P. Factorial structure of the Chinese version of the 12-item General Health Questionnaire in adolescents. J Clin Nurs 2009; 18:3253-61.

22. López-Castedo A, Fernández L. Psychometric properties of the Spanish version of the 12-item General Health Questionnaire in adolescents. Percept Mot Skills 2005; 100:676-80.

23. Worsley A, Gribbin CC. A factor analytic study of the twelve item general health questionnaire. Aust N Z J Psychiatry 1977; 11:269-72.

24. Goldberg DP, Williams P. A user's guide to the General Health Questionnaire. Basingstoke: NferNelson; 1988.

25. Kilic C, Rezaki M, Rezaki B, Kaplan I, Ozgen G, Sagduyu A, et al. General Health Questionnaire (GHQ12 \& GHQ28): psychometric properties and factor structure of the scales in a Turkish primary care sample. Soc Psychiatry Psychiatr Epidemiol 1997; 32:327-31.

26. Martin AJ. Assessing the multidimensionality of the 12-item General Health Questionnaire. Psychol Rep 1999; 84:927-35.

27. Doi Y, Minowa M. Factor structure of the 12-item General Health questionnaire in the Japanese adult population. Psychiatry Clin Neurosci 2003; 57:379-83.

28. Kalliath TJ, O'Driscoll MP, Brough P. A confirmatory factor analysis of the General Health Questionnaire-12. Stress Health 2004; 20:11-20.
29. Andrich D, van Schoubroeck L. The General Health Questionnaire: a psychometric analysis using latent trait theory. Psychol Med 1989; 19:469-85.

30. Montazeri A, Harirchi A, Shariati M, Garmaroudi G, Ebadi M, Fateh A. The 12-item General Health Questionnaire (GHQ-12): translation and validation study of the Iranian version. Health Qual Life Outcomes 2003; 1:66.

31. Shevlin M, Adamson G. Alternative factor models and factorial invariance of the GHQ-12: a large sample analysis using confirmatory factor analysis. Psychol Assess 2005; 17:231-6.

32. Guerje O. Reliability and the factor structure of the Yoruba version of the 12-item General Health Questionnaire. Acta Psychiatr Scand 1991; 84: 125-9.

33. Picardi A, Abeni D, Pasquini P. Assessing psychological distress in patients with skin diseases: reliability, validity and factor structure of the GHQ-12. J Eur Acad Dermatol 2001; 15:410-7.

34. Daradkeh TK, Ghubash R, El-Rufaie EF. Reliability, validity, and factor structure of the Arabic version of the 12-item General Health Questionnaire. Psychol Rep 2001; 89:85-94.

35. Farrell GA. The mental health of hospital nurses in Tasmania as measured by the 12-item Genera Health Questionnaire. J Adv Nurs 1998; 28:707-12.

36. Campbell A, Knowles S. A confirmatory factor analysis of the GHQ-12 using a large Australian sample. Eur J Psychol Assess 2007; 23:2-8.

37. Gouveia VV, Lima TJS, Gouveia RSV, Freires LA, Barbosa LHGM. Questionário de Saúde Geral (QSG-12): o efeito de itens negativos em sua estrutura fatorial. Cad Saúde Pública 2012; 28:375-84.

38. Rey J, Abad F, Barrada J, Ponsoda V. The impact of ambiguous response categories on the factor structure of the GHQ-12. Psychol Assess 2014; 26:1021-30.

39. Molina J, Rodrigo M, Losilla J, Vives J. Wording effects and the factor structure of the 12-Item General Health. Psychol Assess 2014; 26:1031-7.

40. Aguado J, Campbell A, Ascaso C, Navarro P, Garcia-Esteve L, Luciano JV. Examining the factor structure and discriminant validity of the 12 -item General Health Questionnaire (GHQ-12) among Spanish postpartum women. Assessment 2012; 19:517-25.

Recibido el 29/Jun/2013

Versión final presentada el 06/Ene/2015

Aprobado el 21/Ene/2015 